\title{
The jet detection in radio-loud narrow-line Seyfert 1 galaxies
}

\author{
Minfeng Gu \\ Key Laboratory for Research in Galaxies and Cosmology, Shanghai Astronomical Observatory, \\ Chinese Academy of Sciences, 80 Nandan Road, Shanghai, 200030, China \\ email: gumf@shao.ac.cn
}

\begin{abstract}
With relatively small black hole masses and high accretion rates, narrow-line Seyfert 1 galaxies are thought to be young AGNs. About $7 \%$ of them are radio-loud narrow-line Seyfert 1 galaxies (RLNLS1s). RLNLS1s allow us to re-address some of the key questions regarding the physics of jet formation. As the first step of the systematic study on the jet properties of RLNLS1s, we present the radio structure of fourteen RLNLS1s from VLBA observations at 5 $\mathrm{GHz}$ in 2013. Although all these sources are very radio-loud with $R>100$, their jet properties are diverse, in terms of their pc-scale morphology and overall radio spectral shape. The core brightness temperatures of our sources are significantly lower than those of blazars, therefore, the beaming effect is generally not significant, compared to blazars. This implies that the bulk jet speed may likely be low in our sources.
\end{abstract}

Keywords. galaxies: active, galaxies: jets, galaxies: Seyfert, radio continuum: galaxies.

\section{Introduction}

Narrow-line Seyfert 1 galaxies (NLS1s) are a subclass of active galactic nuclei (AGNs), with strong permitted optical/UV Fe II emission lines, relatively weak forbidden-line emission (i.e., [OIII] $5007 / \mathrm{H} \beta<3$ ), and their broad lines are narrower than those of normal broad-line Seyfert 1 galaxies with $\operatorname{FWHM}(\mathrm{H} \beta)$ less than $2000 \mathrm{~km} \mathrm{~s}^{-1}$ (Osterbrock \& Pogge, 1987; Goodrich et al. 1989). NLS1s are often thought to be young and still evolving AGNs with relatively small black hole masses and high accretion rates (see review by Komossa 2008). NLS1s as a class show a low probability to be radio loud at $\sim 7 \%$, compared to normal broad line AGNs (e.g., Komossa et al., 2006; Zhou et al. 2006). Interestingly, radio-loud NLS1s (RLNLS1s) are inhomogeneous in their radio properties. As shown in Komossa et al. (2006), most RLNLS1s in their sample are compact, steep spectrum sources, and hence likely associated with compact steep-spectrum (CSS) radio sources, while only a few NLS1s showed blazar-like behaviour. On the other hand, observational evidence has recently shown that a significant fraction of RLNLS1s at the highest radio-loudnesses, does display the characteristics of blazars, including largeamplitude radio flux and spectral variability, compact radio cores, very high variability brightness temperatures, enhanced optical continuum emission, flat X-ray spectra, and blazar-like spectral energy distributions (e.g., Yuan et al. 2008). RLNLS1s are at an opposite end of AGN correlation space than classical radio-loud AGNs (e.g., Sulentic et al. 2008), in terms of small black hole masses, high accretion rate, and very strong Fe II emission (Komossa et al. 2006). Therefore, RLNLS1s allow us to re-address some of the key questions regarding the physics of jet formation, for example, the physical conditions under which a jet can be launched. 


\section{The jet properties of RLNLS1s}

Relativistic jets were shown to exist in a few RLNLS1s using Very Long Baseline Interferometry (VLBI) observations, based on their high brightness temperatures, and their inverted radio spectra (Doi et al. 2007), which was later confirmed by the detection of flaring $\gamma$-ray emission (Abdo et al. 2009a,b). The previous work based on VLBA data for a few sources argued that RLNLS1s can be either intrinsically radio loud, or apparently radio loud due to jet beaming effects (Gu \& Chen 2010; Doi et al. 2011). These observational results may be contrary to the well-known paradigm that jets are generally associated with elliptical host galaxies in typical radio-loud AGNs, while there is tentative evidence suggesting that at least a few low-redshift RLNLS1s are hosted by spiral galaxies (e.g., Zhou et al. 2007; Antón et al. 2008; León Tavares et al. 2014). Although a number of RLNLS1s have been imaged with VLBI (Gu \& Chen 2010; Doi et al. 2011; Giroletti et al. 2011; D'Ammando et al. 2013b; Wajima et al. 2014; Richards \& Lister 2015), comprehensive large-sample studies of their jet properties on parsec scales have just started ( $\mathrm{Gu}$ et al. 2015; Gu et al. 2016).

\section{1. $\gamma$-ray detected sources}

Nine NLS1s have been detected in the $\gamma$-ray band by the Fermi/LAT with high significance hitherto $^{\dagger}$ (PMN J0948+0022, Abdo et al. 2009a; PKS 1502+036, 1H 0323+342 and PKS 2004-447, Abdo et al. 2009b; SBS 0846+513, D'Ammando et al. 2012; FBQS J1644+2619, D'Ammando et al. 2015; B3 1441+476, Liao et al. 2015; SDSS J122222.55+041315.7, Yao et al. 2015; SDSS J124634.65+023809.0, D’Ammando et al. 2016). All these $\gamma$-ray NLS1s possess a one-sided jet extending from a dominant central core, reminiscent of blazars (e.g., D'Ammando et al., 2013a; Wajima et al., 2014), except for SDSS J124634.65+023809.0, in which only a compact core is detected (see Fig. 1, and also $\mathrm{Gu}$ et al. 2015). The brightness temperature is usually high, well in excess of the equipartition limit and in several cases above the inverse-Compton limit (Schulz et al. 2016). The values are comparable to those of blazars, strongly favoring the beaming effect in these gamma-ray objects. So far, the apparent superluminal motion has been detected in three objects, SBS 0846+513 $\left(\beta_{\text {app }}=(9.3 \pm 0.6) c\right.$, D'Ammando et al. $\left.2013 \mathrm{~b}\right)$, PMN J0948+0022 and 1H 0323+342 (Lister et al. 2016). A maximum apparent speed of $0.94 c$ is reported for SDSS J122222.55+041315. $7^{\ddagger}$, the $\gamma$-ray NLS1 with largest redshift $(z=0.966$ ) (Yao et al., 2015). B3 1441+476, the only NLS1 galaxy both detected in $\gamma$ ray band with high significance and with radio properties similar to CSS sources $\$$ (Liao et al. 2015), shows a core-jet structure with jet extending to about 30 milliarcsecond ( $\mathrm{Gu}$ et al. 2015). The core brightness temperature is $\sim 10^{10.3} \mathrm{~K}$ at $5 \mathrm{GHz}$. The value is at least one order of magnitude lower than most flat-spectrum $\gamma$-ray NLS1 galaxies, indicating intrinsic differences in Doppler boosting of the jet emission.

\subsection{The systematic studies}

In order to understand the jet formation in RLNLS1s, the first systematic investigation on the jet properties based on VLBA observations of 14 sources has been carried out (Gu et al. 2015). All fourteen NLS1 galaxies were detected as single unresolved sources

$\dagger$ Two more tentative candidates, FBQS J1102+2239 and RX J2314.9+2243 were mentioned by Foschini(2011) and Komossa et al. (2015).

$\ddagger$ http://www.physics.purdue.edu/astro/MOJAVE/sourcepages/1219+044.shtml

ฯ The CSS-like NLS1 galaxy, PKS 2004-447, was also detected by Fermi/LAT, however, the optical spectral classification as a NLS1 galaxy continues to remain uncertain.

\| However, the core identification is uncertain ( $\mathrm{Gu}$ et al. 2015). 

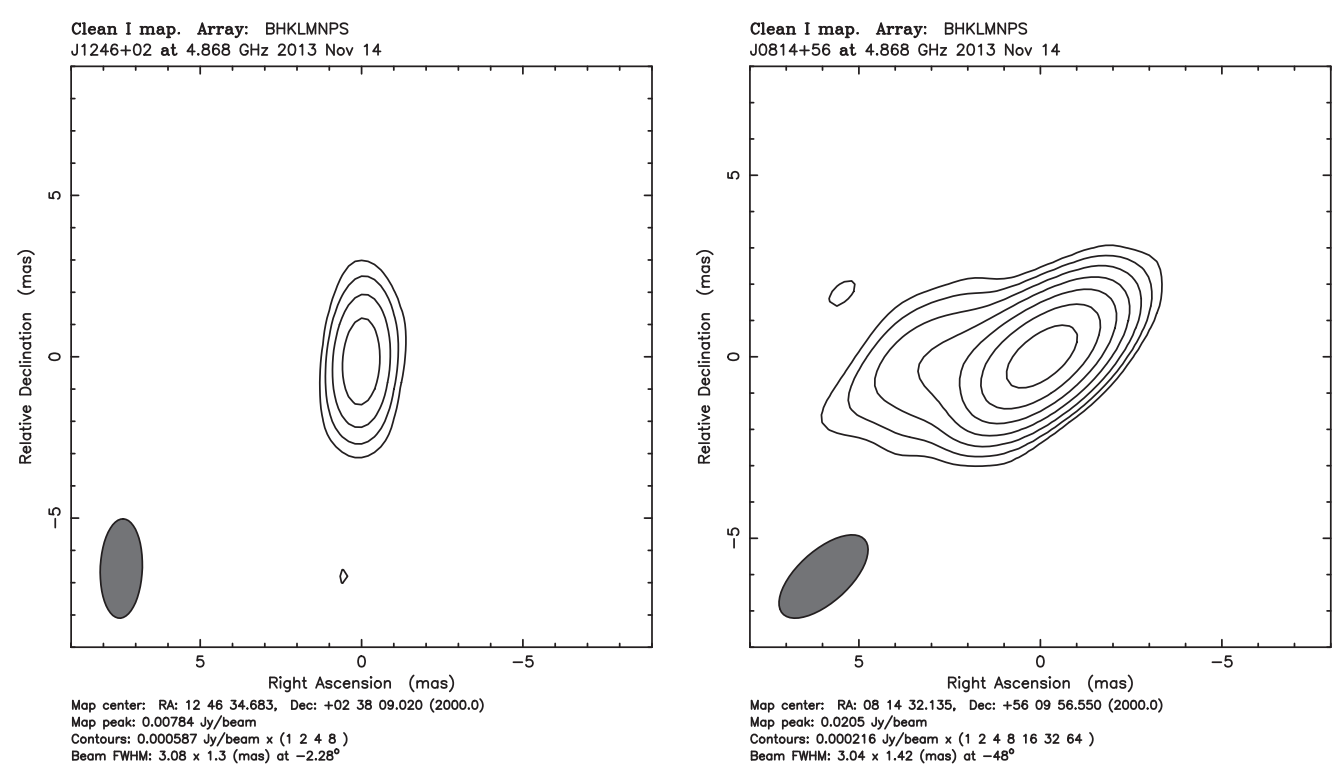

Figure 1. Example VLBA 5 GHz images. Left: SDSS J124634.65+023809.0 with a compact core only. Right: SDSS J081432.11+560956.6 with a core-jet structure.

within 1 arcmin of the SDSS position in both FIRST and NVSS, indicating compact radio structure at FIRST resolution ( 5 arcsec). Although all these sources are very radio-loud with $R>100$, their jet properties are diverse, in terms of their milli-arcsecond (mas) scale (pc scale) morphology and their overall radio spectral shape. From VLBA 5 GHz images, seven sources show a compact core only, while the remaining seven objects have core-jet structure (see example images in Fig. 1). The majority of these sources is compact at VLBI mas scales with all components being within $\sim 5$ mas $(\sim 24-38$ pc depending on source redshift). In combination with archival multi-frequency data, seven sources show flat or even inverted radio spectra $\left(\alpha \leqslant 0.5, f_{\nu} \propto \nu^{-\alpha}\right)$, while steep spectra $(\alpha>0.5)$ are revealed in the remaining seven objects.

From the high-resolution VLBA images, the brightness temperature of the radio core in the rest frame can be estimated (e.g., Ghisellini et al. 1993), which is often used to constrain the jet Doppler factor in combination with the assumed intrinsic brightness temperature, either as the equipartition brightness temperature of $5 \times 10^{10} \mathrm{~K}$ (Readhead 1994), or the inverse Compton catastrophe brightness temperature of $\sim 10^{12} \mathrm{~K}$ (Kellermann \& Pauliny-Toth 1969). The core brightness temperature ranges from $10^{8.4}$ to $10^{11.4} \mathrm{~K}$ with a median value of $10^{10.1} \mathrm{~K}$. This indicates that the radio emission is from non-thermal jets, confirming that powerful jets can be formed in accretion systems with relatively small black hole masses and high accretion rates ( $\mathrm{Gu} \&$ Chen 2010, Doi et al. 2011). However, the core brightness temperatures of these sources are significantly lower than those of blazars, as the VLBA core brightness temperatures of blazars typically range between $10^{11}$ and $10^{13} \mathrm{~K}$ with a median value near $10^{12} \mathrm{~K}$, and can even extend up to $5 \times 10^{13} \mathrm{~K}$ (Kovalev et al. 2005, 2009). The values are also well below those of flat-spectrum $\gamma$-ray NLS1 galaxies. Therefore, the beaming effect is generally not significant in these sources, implying that the bulk jet speed may likely be low. The evidence for slow jet speeds (i.e., less relativistic jets), in combination with the low kinetic/radio power, may offer an explanation for the compact VLBA radio structure in most sources. The mildly relativistic jets in these high accretion rate systems are consistent with a 
scenario, where jets are accelerated from the hot corona above the disk by the magnetic field and the radiation force of the accretion disk (Cao 2014). Alternatively, a low jet bulk velocity can be explained by low spin in the Blandford-Znajek mechanism (Blandford \& Znajek 1977).

\section{Acknowledgements}

This work is supported by the National Science Foundation of China (grants 11473054 and U1531245) and by the Science and Technology Commission of Shanghai Municipality (grant 14ZR1447100).

\section{References}

Abdo, A. A., et al. 2009a, ApJ, 699, 976

Abdo, A. A., et al. 2009b, ApJ, 707, L142

Antón, S., Browne, I. W. A., \& Marchã, M. J. 2008, A\&A, 490, 583

Blandford, R. D. \& Znajek, R. L. 1977, MNRAS, 179, 433

Cao, X. 2014, ApJ, 783, 51

D'Ammando, F., Orienti, M., Finke, J., et al. 2012, MNRAS, 426, 317

D'Ammando, F., Orienti, M., Doi, A., et al. 2013a, MNRAS, 433, 952

D'Ammando, F., Orienti, M., Finke, J., et al. 2013b, MNRAS, 436, 191

D'Ammando, F., Orienti, M., Larsson, J., \& Giroletti, M. 2015, MNRAS, 452, 520

D'Ammando, F., Orienti, M., Finke, J., et al. 2016, arXiv:1609.04434

Doi, A., et al. 2007, PASJ, 59, 703

Doi, A., Asada, K., \& Nagai, H. 2011, ApJ, 738, 126

Foschini, L. 2011, Narrow-Line Seyfert 1 Galaxies and their Place in the Universe, 24

Ghisellini, G., Padovani, P., Celotti, A., \& Maraschi, L. 1993, ApJ, 407, 65

Giroletti, M., Paragi, Z., Bignall, H., et al. 2011, A\&AA, 528, L11

Goodrich, R. W. 1989, ApJ, 342, 22

Gu, M. \& Chen, Y. 2010, AJ, 139, 2612

Gu, M., Chen, Y., Komossa, S., et al. 2015, ApJS, 221, 3

Gu, M., Chen, Y., Komossa, S., Yuan, W., \& Shen, Z. 2016, Astronomische Nachrichten, 337, 125

Kellermann, K. I., Pauliny-Toth I. I. K. 1969, ApJ, 155, L71

Komossa, S., Voges, W., Xu, D., Mathur, S., Adorf, H. M., Lemson, G., Duschl, W., \& Grupe D. $2006, A J, 132,531$

Komossa, S. 2008, Rev. Mex. AA Ser. Conf., 32, 86

Komossa, S., Xu, D., Fuhrmann, L., et al. 2015, A\&A, 574, A121

Kovalev, Y. Y., Kellermann, K. I., Lister, M. L., et al. 2005, AJ, 130, 2473

Kovalev, Y. Y., Aller, H. D., Aller, M. F., et al. 2009, ApJL, 696, L17

León Tavares, J., Kotilainen, J., Chavushyan, V., et al. 2014, ApJ, 795, 58

Liao, N.-H., Liang, Y.-F., Weng, S.-S., Gu, M.-F., \& Fan, Y.-Z. 2015, arXiv:1510.05584

Lister, M. L., Aller, M. F., Aller, H. D., et al. 2016, AJ, 152, 12

Osterbrock D. E., Pogge R. W. 1985, ApJ, 297, 166

Readhead, A. C. S.. 1994, ApJ, 426, 51

Richards, J. L. \& Lister, M. L. 2015, ApJL, 800, L8

Schulz, R., Kreikenbohm, A., Kadler, M., et al. 2016, A\&\&A, 588, A146

Sulentic, J. W., Zamfir, S., Marziani, P., \& Dultzin, D. 2008, Revista Mexicana de Astronomia y Astrofisica Conference Series, 32, 51

Wajima, K., Fujisawa, K., Hayashida, M., et al. 2014, ApJ, 781, 75

Yao, S., Yuan, W., Komossa, S., et al. 2015, AJ, 150, 23

Yuan, W., Zhou, H. Y., Komossa, S., Dong, X. B., Wang T. G., Lu, H. L., \& Bai J. M. 2008, ApJ, 685, 801

Zhou, H., Wang, T., Yuan, W., Lu, H., Dong, X., Wang, J., \& Lu Y. 2006, ApJS, 166, 128

Zhou, H., et al., 2007, ApJ, 658, L13 